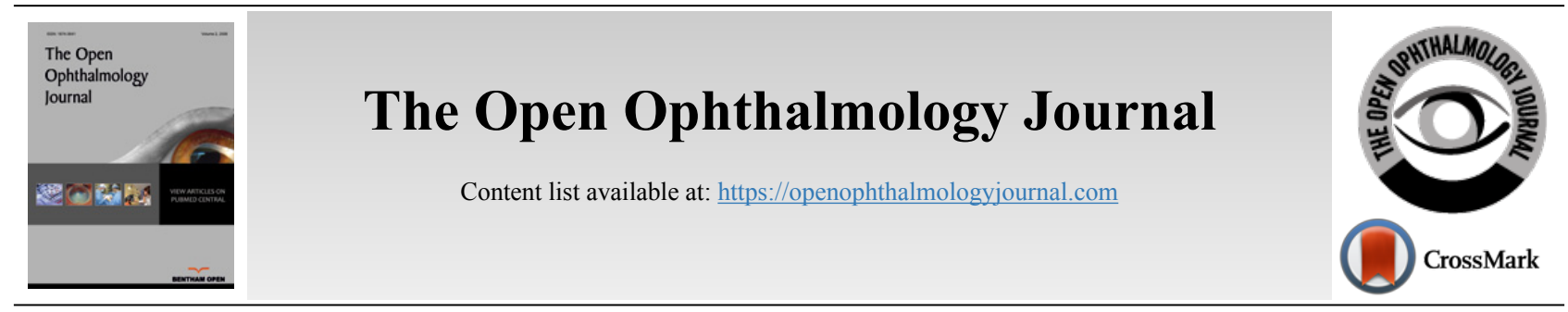

RESEARCH ARTICLE

\title{
Knowledge, Attitudes, and Practices of Optometrists Regarding Low Vision Services in Saudi Arabia
}

\author{
Godwin O. Ovenseri-Ogbomo ${ }^{1, *(D)}$ and Waleed Alghamdi ${ }^{1(D)}$ \\ ${ }^{1}$ Department of Optometry, College of Applied Medical Sciences, Qassim University, Saudi Arabia
}

\begin{abstract}
:
Purpose:

To address the human resources challenge for the provision of low vision services in Saudi Arabia, this study sought to investigate the knowledge, attitudes, and practices of optometrists in Saudi Arabia regarding low vision services. The knowledge and attitudes to low vision services can influence the provision of low vision services by optometrists.

\section{Methods:}

A prospective cross-sectional survey of optometrists practicing in Saudi Arabia was undertaken using an online questionnaire designed to elicit the opinions of respondents. The online questionnaire was sent out to optometrists on the official mailing list of registered optometrists and those on the mailing list of the Saudi Society of Optometry.

Results:

Only $26.5 \%$ of the respondents correctly indicated the correct designation of low vision in terms of visual acuity. Although $95.8 \%$ indicated that optical low vision devices could help people with low vision, $81.6 \%$ reported that low vision devices were expensive, and $42.9 \%$ felt low vision practice was not profitable. Only $10.4 \%$ of respondents provide low vision services in their practice. Insufficient training in low vision care was the main barrier militating against the provision of low vision services.

Conclusion:

The pertinent finding in this study is that about a quarter of the respondents could correctly designate low vision in terms of visual acuity using the World Health Organization (WHO) definition. The study concluded that there was poor knowledge, attitudes, and practices of optometrists in Saudi Arabia regarding low vision, which has implications for the provision of low vision services by optometrists.
\end{abstract}

Keywords: Low vision practice, Optometrists, Saudi Arabia, Perception, Barriers, Vision impairment.

Article History

Received: January 23, 2021

Revised: July 17, 2021

Accepted: July 28, 2021

\section{INTRODUCTION}

Visual impairment is a major public health challenge and has implications for the individual affected, the family, and society. According to the World Health Organization (WHO), visual impairment encompasses both low vision and blindness, with both defined in terms of visual acuity loss. Visual acuity of less than $6 / 18$ but equal to or better than $3 / 60$ and a visual field loss of less than 20 characterizes low vision, while blindness is a visual acuity of less than $3 / 60$ and a visual field loss of less than $10[1,2]$. Low vision has a significant

\footnotetext{
* Address correspondence to this author at Department of Optometry, College of Applied Medical Sciences, Qassim University, Saudi Arabia;

Tel: +966592230573;

E-mails: godwin.ovenseri-ogbomo@uniben.edu; g.ovenseri@qu.edu.sa
}

economic, social, psychological, and educational impact on those affected. The provision of low vision services to affected individuals could mitigate some of these impacts.

Low vision is a significant public health concern in the Kingdom of Saudi Arabia (KSA). A nationwide survey of blindness reported the prevalence of blindness and low vision to be $1.5 \%$ and $7.8 \%$, respectively [3]. This was reported to be 20 times the magnitude of blindness in the United States [4]. Regional variation exists in the prevalence of low vision and visual impairment across KSA - ranging from $0.7 \%$ in Bisha to $6.4 \%$ in Asha $[5-8]$. There are also age variations in the prevalence of visual impairment in KSA. In adults 18 years and older, the prevalence of visual impairment has been reported to be $13.9 \%$ in Aljouf province [3] and 23.5\% in Arar district [9], 
whereas in children younger than 18 years, the prevalence of visual impairment is $7.0 \%$ in Riyadh [10] and $8.8 \%$ in Al-Baha region [11].

Given the magnitude of low vision and visual impairment in the Kingdom, there appears to be poor low vision service provision. In a study of the state of low vision in 2006 [12], it was reported that $95 \%$ of hospitals in Riyadh do not provide low vision services and that $75 \%$ of low vision cases were seen by ophthalmologists while optometrists see $25 \%$. The author [12] further remarked that many of those who attend to patients with low vision have not had any specialist training in low vision care. This report underscores the dire situation with the provision of low vision services in Saudi Arabia.

In the present study, the authors could not verify the number of optometrists in Saudi Arabia. While the number of optometrists could not be precisely determined, it has been estimated that there is one optometrist for every 95,500 people in Saudi Arabia [13, 14]. This ratio falls short of the best practice in developed countries such as the USA and Australia $(1: 10,000)[14]$ or the minimum recommended by the World Health Organization (WHO) $(1: 50,000)$ [15].

This shortfall in the number of optometrists will further impact the provision of low vision services in the Kingdom. Following their global survey of low vision services, Chiang and Keeffe [16] suggested three areas for action to improve access to low vision services: human resources, sustainability of services, and advocacy [17]. In order to address the human resource component of the strategy for improving access to low vision services in Saudi Arabia, this study was designed to investigate the knowledge, attitudes, and practices of optometrists in Saudi Arabia regarding low vision services. The information obtained will help in addressing the human resource for low vision services in KSA. Furthermore, the outcome of this study could form a basis for review of the current curriculum of optometrists in Saudi Arabia with the goal of including content that will enable graduates of the training institutions to be able to provide low vision services.

\section{MATERIALS AND METHODS}

This prospective cross-sectional exploratory study was conducted among optometrists practicing in the Kingdom of Saudi Arabia (KSA). The study employed the knowledge, attitudes, and practices (KAP) approach using a semistructured questionnaire to survey the opinion of optometrists in Saudi Arabia. The KAP survey has been reported to be the best approach in a study designed to identify baseline knowledge, attitudes, and practices as well as provide information on needs and barriers related to a subject matter $[18,19]$. A link to the pre-tested questionnaire was e-mailed out to optometrists on the official mailing list of registered optometrists and those on the mailing list of the Saudi Society of Optometry. Furthermore, the link to the online questionnaire was sent via social media such as WhatsApp and Twitter to optometrists in Saudi Arabia. However, only 49 (representing
$6.1 \%$ of the registered optometrists in Saudi Arabia in 2018) [20] optometrists completed and returned their questionnaires. Participants responded to questions about basic demographic information (age, sex, where they had their optometry education etc.), questions relating to their knowledge of low vision, attitudes, and practice as well as referrals for patients with low vision, and questions relating to factors that could serve as barriers to the provision of low vision services. The questions comprised both open- and closed-ended questions. The study was approved by the Research Committee, College of Applied Medical Sciences, Qassim University. The study adhered strictly to the tenets of the Helsinki Declaration on the use of humans for medical research. To ensure participants' confidentiality, the questionnaire was anonymized, and no identifying information was collected.

The responses were collated and analyzed using the Statistical Package for Social Sciences (SPSS) version 24. Descriptive statistics, including frequencies, percentages, means, and standard deviations, were computed. Chi-squared $\left(\chi^{2}\right)$ was used to determine the association between categorical variables. Results were presented as tables and charts where appropriate.

\section{RESULTS}

A total of 49 optometrists practicing in Saudi Arabia responded to the online questionnaire. This comprised 15 (30.6\%) females and $34(69.4 \%)$ males. They were aged $24-$ 50 years with a median age of 30 years. The mean age of the respondents was $31.71 \pm 6.67$ years. The mean age of the males was $33.67 \pm 6.71$ years, while the mean age of the females was $27.80 \pm 4.69$ years. The males were significantly older than the females $(\mathrm{p}=0.004)$.

Of the 49 respondents, 39 (79.6\%) were Saudi nationals while $9(18.3 \%)$ were foreign nationals. Fig. (1) shows the distribution of the respondents by nationality.

A total of 48 (98\%) reported the number of years they had practiced optometry. The respondents have practiced optometry between 1 and 26 years, with $68.8 \%$ of the respondents having been in practice for 10 years. The mean number of years in practice was $7.77 \pm 6.27$ years $(95 \% \mathrm{CI}=$ $5.94-9.59)$. The 34 male respondents had practiced optometry between 1 and 26 years $($ mean $=8.97 \pm 6.51$ years, $95 \% \mathrm{CI}=$ $6.70-11.24$ ), while the 14 female respondents had practiced between 1 and 14 years $($ mean $=4.86 \pm 4.64$ years, $95 \% \mathrm{CI}=$ $2.18-7.54)$. The males have been in practice longer than the females $(\mathrm{p}=0.019)$.

Fig. (2) shows the mode of practice of the 49 respondents, with $51 \%$ practicing in government hospitals, including military hospitals. $24(49 \%)$ of the respondents had additional training in low vision beyond their undergraduate optometry training. This included $5(20.8 \%)$ who have higher degrees and post-graduation diploma and $19(79.2 \%)$ who attended workshops in low vision as part of continuing professional training. 


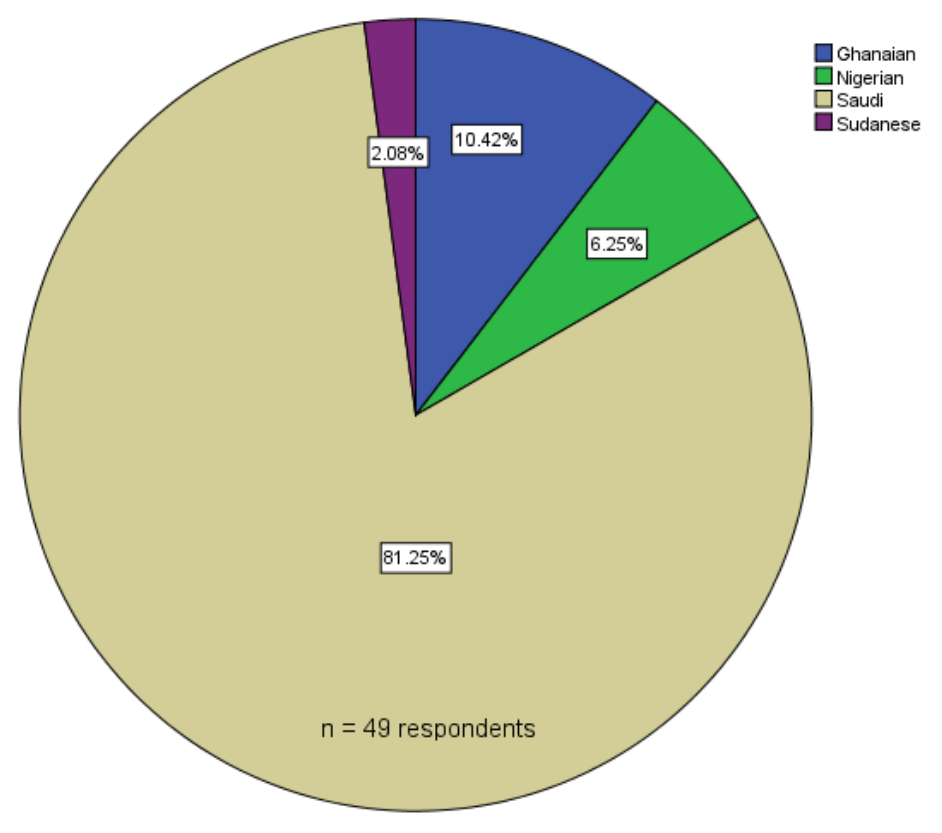

Fig. (1). Distribution of respondents according to their nationality.

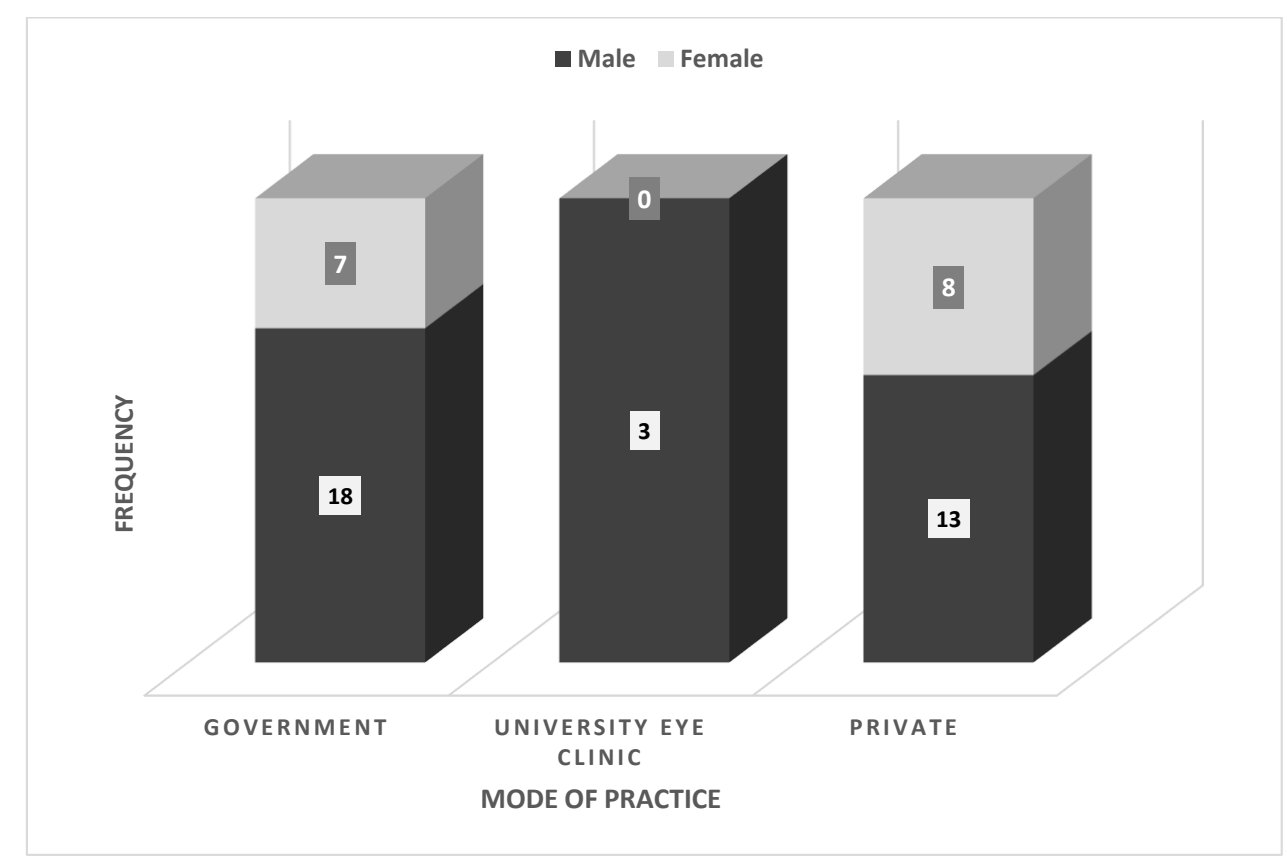

Fig. (2). Mode of the practice of respondents. Private include optical shops and private hospitals.

\subsection{Knowledge of Low Vision}

Table 1 shows the knowledge of low vision reported by the respondents. Only $13(26.5 \%)$ of the respondents correctly indicated the 2 designation of low vision in terms of visual acuity (VA). Furthermore, 19 (38.8\%) correctly reported that low vision is defined after medical, surgical, and optical correction of reduced vision. On the other hand, 34 (69.4\%) of the respondents correctly indicated that low vision is present in spite of visual acuity if there is constricted visual field.
Univariate analysis indicated that the knowledge of low vision in terms of visual acuity, visual field, and whether the low vision is considered to be present after the medical, surgical, and optical correction was not influenced by gender, place where optometry training was obtained, or whether respondents have had any low vision training post-graduation. Whether a respondent had correct knowledge of low vision in terms of visual acuity was significantly related to the mode of practice $(\mathrm{p}=0.023)($ Table 2$)$. 
Table 1. Knowledge of definition of low vision.

\begin{tabular}{|c|c|c|}
\hline Item & Frequency & Percentage \\
\hline \multicolumn{3}{|l|}{ VA cut of low vision } \\
\hline $6 / 12$ or worse & 2 & 4.1 \\
\hline $6 / 18$ or worse & 13 & 26.5 \\
\hline $6 / 60$ or worse & 33 & 67.3 \\
\hline Any reduction in VA & 1 & 2.0 \\
\hline \multicolumn{3}{|c|}{ POAG* with $6 / 9$ vision but constricted field } \\
\hline Yes & 34 & 69.4 \\
\hline No & 14 & 28.6 \\
\hline Not sure & 1 & 2.0 \\
\hline \multicolumn{3}{|c|}{ Low vision is after medical, surgical, and optical correction } \\
\hline Yes & 19 & 38.8 \\
\hline No & 28 & 57.1 \\
\hline No response & 2 & 4.1 \\
\hline
\end{tabular}

${ }^{*} \mathrm{POAG}=$ primary open-angle glaucoma.

Table 2. Univariate analysis of factors influencing knowledge of low vision.

\begin{tabular}{|c|c|c|c|}
\hline \multicolumn{3}{|c|}{ Characteristics } & \multirow[t]{3}{*}{ p-value } \\
\hline \multicolumn{3}{|c|}{ Correct knowledge of low vision in terms of visual acuity } & \\
\hline & Yes $(n, \%)$ & No $(n, \%)$ & \\
\hline \multicolumn{4}{|l|}{ Gender } \\
\hline Male & $9(26.47)$ & $25(73.53)$ & \multirow[t]{2}{*}{0.989} \\
\hline Female & $4(26.67)$ & $11(73.33)$ & \\
\hline \multicolumn{4}{|l|}{ Mode of practice } \\
\hline Government & $8(66.67)$ & $4(33.33)$ & \multirow[t]{3}{*}{$0.023 *$} \\
\hline University Eye Clinic & $1(33.33)$ & $2(66.67)$ & \\
\hline Private & $4(19.05)$ & $17(80.95)$ & \\
\hline \multicolumn{4}{|c|}{ Place where the respondent had optometry training } \\
\hline Inside Saudi Arabia & $9(24.32)$ & $28(75.68)$ & \multirow[t]{2}{*}{0.430} \\
\hline Outside Saudi Arabia & $4(27.08)$ & $7(63.64)$ & \\
\hline \multicolumn{4}{|c|}{ Low vision training after graduation } \\
\hline Yes & $7(29.17)$ & $17(70.83)$ & \multirow[t]{2}{*}{0.682} \\
\hline No & $6(24.00)$ & $19(73.47)$ & \\
\hline \multicolumn{4}{|l|}{ Years in practice } \\
\hline$<10$ years & $6(19.35)$ & $25(80.65)$ & \multirow[t]{2}{*}{0.223} \\
\hline$>10$ year & $6(35.29)$ & $11(64.71)$ & \\
\hline \multicolumn{4}{|c|}{ Correct knowledge of low vision in terms of Visual field } \\
\hline \multicolumn{4}{|c|}{ Gender } \\
\hline Male & $24(70.59)$ & $10(29.41)$ & \multirow[t]{2}{*}{0.784} \\
\hline Female & $10(66.67)$ & $5(33.33)$ & \\
\hline \multicolumn{4}{|l|}{ Mode of practice } \\
\hline Government & $17(68.00)$ & $8(32.00)$ & \multirow[t]{3}{*}{0.314} \\
\hline University Eye Clinic & $1(33.33)$ & $2(66.67)$ & \\
\hline Private & $16(76.19)$ & $5(23.81)$ & \\
\hline \multicolumn{4}{|c|}{ Place where the respondent had optometry training } \\
\hline Inside Saudi Arabia & $25(67.57)$ & $12(32.43)$ & \multirow[t]{2}{*}{0.747} \\
\hline Outside Saudi Arabia & $8(72.75)$ & $3(27.27)$ & \\
\hline \multicolumn{4}{|c|}{ Low vision training after graduation } \\
\hline Yes & $16(47.06)$ & $18(52.94)$ & \multirow[t]{2}{*}{0.686} \\
\hline No & $8(53.33)$ & $7(46.67)$ & \\
\hline Years in practice & & & \\
\hline
\end{tabular}


(Table 2) contd.....

\begin{tabular}{|c|c|c|c|}
\hline \multicolumn{3}{|c|}{ Characteristics } & \multirow{3}{*}{$\frac{\text { p-value }}{0.133}$} \\
\hline$<10$ years & $24(77.42)$ & $7(22.58)$ & \\
\hline$>10$ years & $9(56.25)$ & $7(43.75)$ & \\
\hline \multicolumn{4}{|c|}{ Correct knowledge of low vision in terms of definition after treatment } \\
\hline \multicolumn{4}{|c|}{ Gender } \\
\hline Male & $19(59.38)$ & $13(40.63)$ & \multirow[t]{2}{*}{0.968} \\
\hline Female & $9(60.00)$ & $6(40.00)$ & \\
\hline \multicolumn{4}{|l|}{ Mode of practice } \\
\hline Government & $10(43.48)$ & $13(56.52)$ & \multirow[t]{3}{*}{0.057} \\
\hline University Eye Clinic & $3(100.00)$ & $0(0.00)$ & \\
\hline Private & $15(71.43)$ & $6(28.57)$ & \\
\hline \multicolumn{4}{|c|}{ Place where the respondent had optometry training } \\
\hline Inside Saudi Arabia & $20(57.14)$ & $15(42.86)$ & \multirow[t]{2}{*}{0.356} \\
\hline Outside Saudi Arabia & $8(72.73)$ & $3(27.27)$ & \\
\hline \multicolumn{4}{|c|}{ Low vision training after graduation } \\
\hline Yes & $12(52.17)$ & $11(47.83)$ & \multirow[t]{2}{*}{0.312} \\
\hline No & $16(66.67)$ & $8(33.33)$ & \\
\hline \multicolumn{4}{|c|}{ Year in practice } \\
\hline$<10$ years & $19(63.33)$ & $11(36.67)$ & \multirow[t]{2}{*}{0.639} \\
\hline$>10$ years & $9(56.25)$ & $7(43.75)$ & \\
\hline
\end{tabular}

The respondents reported age-related macular degeneration (ARMD), glaucoma, and retinitis pigmentosa as leading causes of low vision. Curiously, keratoconus, conjunctivitis, and dry eye disease were also reported as causes of low vision (Fig. 3).

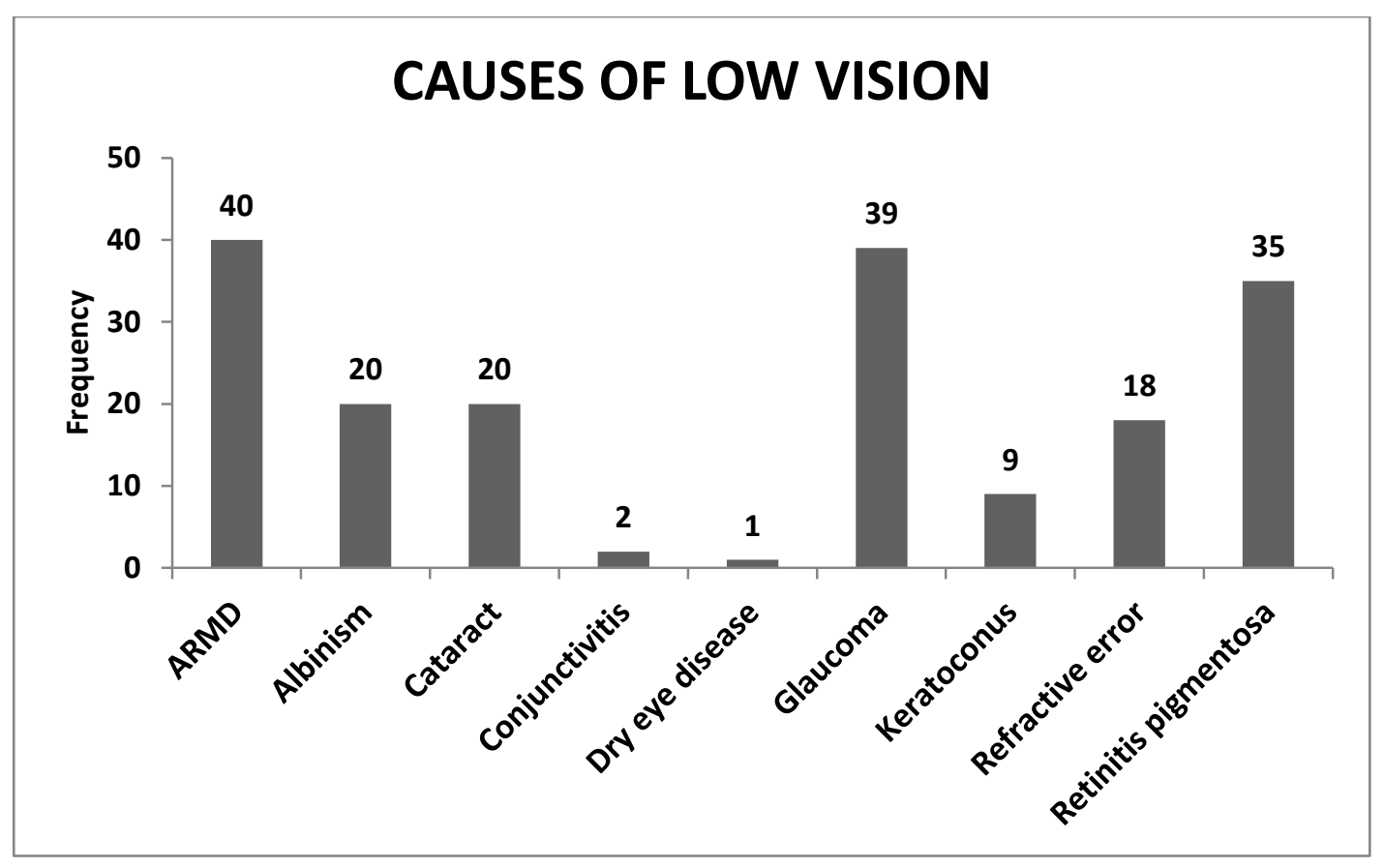

Fig. (3). Cause of low vision reported by respondents. (Total frequency is more than the number of respondents because of multiple responses).

\subsection{Attitude to Low Vision}

The attitude of respondents to low vision is shown in Table 3. As much as $45(91.8 \%)$ of the respondents indicated that patients with low vision are not blind. Therefore, something can be done to improve their vision. Consequently 46 (95.8\%) indicated that optical low vision devices help low vision patients to improve visual functioning. These were considered to be positive attitudes. On the contrary, $40(81.6 \%)$ of the respondents indicated that low vision devices were expensive, while $21(42.9 \%)$ reported that low vision practice was not profitable. These were considered to be negative attitudes.

Univariate analysis of factors influencing respondents' attitude to low vision is shown in Table 4. The perception that optical low vision devices are expensive was significantly associated with whether respondents obtained their training inside or outside Saudi Arabia $(\mathrm{p}=0.035)$. 
Table 3. Respondents' attitude to low vision.

\begin{tabular}{|c|c|c|}
\hline Item & Frequency & \multicolumn{2}{|c|}{ Percentage } \\
\hline Patients with low vision are blind, so nothing much can be done & 91.8 \\
\hline False & 45 & 2.0 \\
\hline True & 1 & 6.1 \\
\hline I don't know Low vision practice is time-consuming & 3 & 57.1 \\
\hline False & 28 & 30.6 \\
\hline True & 15 & 12.2 \\
\hline Not sure & 6 & 95.8 \\
\hline False & & 4.1 \\
\hline True & 46 & 2.0 \\
\hline No response & 2 & \\
\hline LVDs are expensive & 1 & 10.2 \\
\hline False & & 81.6 \\
\hline True & 5.2 \\
\hline Not sure & 5 & \\
\hline False & 40 & 34.7 \\
\hline True & 4 & 42.9 \\
\hline Not sure & & 22.4 \\
\hline
\end{tabular}

*LVDs $=$ low vision devices.

Table 4. Univariate analysis of factors influencing the attitude.

\begin{tabular}{|c|c|c|c|}
\hline \multicolumn{3}{|c|}{ Characteristics } & \multirow[t]{3}{*}{ p-value } \\
\hline \multicolumn{3}{|c|}{ People with low vision are blind, and so nothing can be done to help } & \\
\hline & False (n, \%) & True (n, \%) & \\
\hline \multicolumn{4}{|l|}{ Gender } \\
\hline Male & $32(96.97)$ & $1(3.03)$ & \multirow[t]{2}{*}{0.526} \\
\hline Female & $13(100.00)$ & $0(0.00)$ & \\
\hline \multicolumn{4}{|l|}{ Mode of practice } \\
\hline Government & $22(95.65)$ & $1(4.35)$ & \multirow[t]{3}{*}{0.929} \\
\hline University Eye Clinic & $3(100.00)$ & $0(0.00)$ & \\
\hline Private & $20(95.24)$ & $1(4.76)$ & \\
\hline \multicolumn{4}{|c|}{ Place where the respondent had optometry training } \\
\hline Inside Saudi Arabia & $34(97.14)$ & $1(2.86)$ & \multirow[t]{2}{*}{0.377} \\
\hline Outside Saudi Arabia & $10(90.91)$ & $1(9.09)$ & \\
\hline \multicolumn{4}{|c|}{ Low vision training after graduation } \\
\hline Yes & $23(100.00)$ & $0(0.00)$ & \multirow[t]{2}{*}{0.312} \\
\hline No & $22(95.65)$ & $1(2.17)$ & \\
\hline \multicolumn{4}{|l|}{ Years in practice } \\
\hline$<10$ years & $27(96.43)$ & $1(3.57)$ & \multirow[t]{2}{*}{0.431} \\
\hline$>10$ year & $17(100.00)$ & $0(0.00)$ & \\
\hline \multicolumn{4}{|c|}{ Low vision practice is time consuming } \\
\hline \multicolumn{4}{|c|}{ Gender } \\
\hline Male & $9(30.00)$ & $21(70.00)$ & \multirow[t]{2}{*}{0.307} \\
\hline Female & $6(46.15)$ & $7(53.85)$ & \\
\hline \multicolumn{4}{|l|}{ Mode of practice } \\
\hline Government & $7(31.82)$ & $15(68.18)$ & \multirow[t]{3}{*}{0.895} \\
\hline University Eye Clinic & $1(33.33)$ & $2(66.67)$ & \\
\hline Private & $7(38.89)$ & $11(61.11)$ & \\
\hline Place where the respondent had opton & & & \\
\hline
\end{tabular}


(Table 4) contd.....

\begin{tabular}{|c|c|c|c|}
\hline \multicolumn{3}{|c|}{ Characteristics } & \multirow{3}{*}{$\frac{\text { p-value }}{>0.999}$} \\
\hline Inside Saudi Arabia & $11(33.33)$ & $22(66.67)$ & \\
\hline Outside Saudi Arabia & $3(33.33)$ & $6(66.67)$ & \\
\hline \multicolumn{4}{|c|}{ Low vision training after graduation } \\
\hline Yes & $8(36.36)$ & $14(63.64)$ & \multirow[t]{2}{*}{0.835} \\
\hline No & $7(33.33)$ & $14(66.67)$ & \\
\hline \multicolumn{4}{|l|}{ Years in practice } \\
\hline$<10$ years & $12(44.44)$ & $15(55.56)$ & \multirow[t]{2}{*}{0.113} \\
\hline$>10$ years & $3(20.00)$ & $12(80.00)$ & \\
\hline \multicolumn{4}{|c|}{ LVDs do not help people with low vision } \\
\hline \multicolumn{4}{|l|}{ Gender } \\
\hline Male & $32(94.12)$ & $2(5.88)$ & \multirow[t]{2}{*}{0.354} \\
\hline Female & $14(100.00)$ & $0(0.00)$ & \\
\hline \multicolumn{4}{|l|}{ Mode of practice } \\
\hline Government & $24(96.00)$ & $1(4.00)$ & \multirow[t]{3}{*}{$0.026^{*}$} \\
\hline University Eye Clinic & $2(66.67)$ & $1(33.33)$ & \\
\hline Private & $20(100.00)$ & $0(0.00)$ & \\
\hline \multicolumn{4}{|c|}{ Place where the respondent had optometry training } \\
\hline Inside Saudi Arabia & $35(97.22)$ & $1(2.78)$ & \multirow[t]{2}{*}{0.364} \\
\hline Outside Saudi Arabia & $10(90.91)$ & $1(9.09)$ & \\
\hline \multicolumn{4}{|c|}{ Low vision training after graduation } \\
\hline Yes & $23(95.83)$ & $1(4.17)$ & \multirow[t]{2}{*}{$>0.999$} \\
\hline No & $23(95.83)$ & $1(4.17)$ & \\
\hline \multicolumn{4}{|l|}{ Year in practice } \\
\hline$<10$ years & $29(96.67)$ & $1(3.33)$ & \multirow[t]{2}{*}{0.677} \\
\hline$>10$ years & $16(94.12)$ & $1(5.88)$ & \\
\hline \multicolumn{4}{|c|}{ Optical low vision devices are expensive } \\
\hline \multicolumn{4}{|c|}{ Gender } \\
\hline Male & $3(9.68)$ & $28(90.32)$ & \multirow[t]{2}{*}{0.649} \\
\hline Female & $2(14.29)$ & $12(85.71)$ & \\
\hline Mode of practice & & & \\
\hline Government & $2(8.70)$ & $21(91.30)$ & 0.440 \\
\hline University Eye Clinic & $1(33.33)$ & $2(66.67)$ & \\
\hline Private & $2(10.53)$ & $17(89.47)$ & \\
\hline & tometry train & & \\
\hline Inside Saudi Arabia & $2(5.88)$ & $32(94.12)$ & $0.035^{*}$ \\
\hline Outside Saudi Arabia & $3(30.00)$ & $7(70.00)$ & \\
\hline Low vision training after gradu & & & \\
\hline Yes & $4(17.39)$ & $19(82.61)$ & 0.170 \\
\hline No & $1(4.55)$ & $21(95.45)$ & \\
\hline Years in practice & & & \\
\hline$<10$ years & $3(10.71)$ & $25(89.29)$ & 0.858 \\
\hline$>10$ years & $2(12.50)$ & $14(87.50)$ & \\
\hline & rofitable & & \\
\hline Gender & & & \\
\hline Male & $14(48.12)$ & $15(51.72)$ & 0.431 \\
\hline Female & $3(33.33)$ & $6(66.67)$ & \\
\hline Mode of practice & & & \\
\hline Government & $7(36.84)$ & $12(63.16)$ & 0.225 \\
\hline University Eye Clinic & $2(100.00)$ & $0(0.00)$ & \\
\hline Private & $8(47.06)$ & $9(52.94)$ & \\
\hline Pla & tometry train & & \\
\hline Inside Saudi Arabia & $10(38.46)$ & $16(61.54)$ & 0.367 \\
\hline Outside Saudi Arabia & $6(54.55)$ & $5(45.45)$ & \\
\hline & caduation & & \\
\hline
\end{tabular}


(Table 4) contd.....

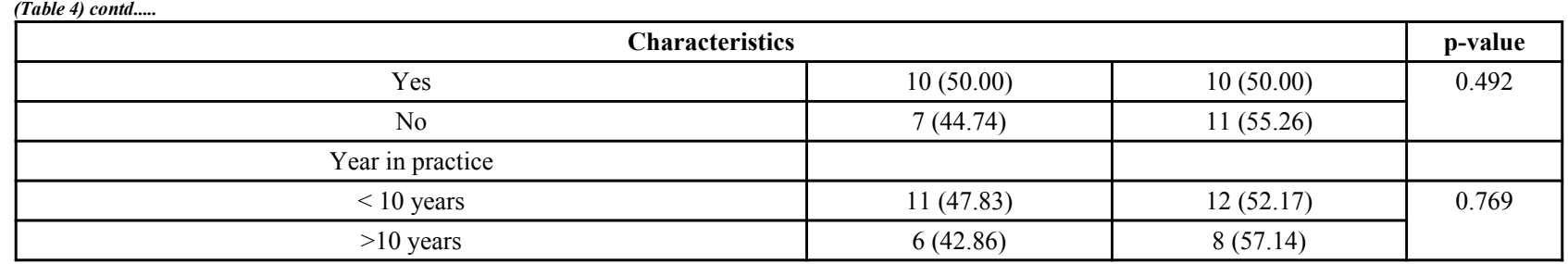

\subsection{Practice Related to Low Vision}

A total of $41(83.7 \%)$ of the respondents have encountered patients with low vision since they started practicing optometry. However, only $10(20.4 \%)$ provide low vision services (LVS) in their clinic/hospital (Table 5). With respect to referral practices, $36(73.5 \%)$ provide referral services for patients with low vision.

Table 6 shows the univariate analysis of the factors influencing the practice of low vision by respondents. Respondents who have practiced for less than 10 years were more likely to refer patients with low vision than those who have practiced for more than 10 years $(\mathrm{OR}=4.04,95 \% \mathrm{CI}=$ 0.83 - 20.20). Furthermore, whether respondents provide low vision services was related to years in practice $(p=0.01)$. Also, optometrists who had their training outside of Saudi Arabia were likely not to know where low vision services are provided in the Kingdom $(\mathrm{p}<0.0001)$.

Table 5. Referral practices with respect to low vision.

\begin{tabular}{|c|c|c|}
\hline Item & Frequency & Percentage \\
\hline \multicolumn{3}{|c|}{ Encounter patients with low vision since you started practicing } \\
\hline Yes & 41 & 83.7 \\
\hline No & 8 & 16.3 \\
\hline \multicolumn{3}{|c|}{ Do you provide low vision service in your clinic/hospital? } \\
\hline Yes & 10 & 20.4 \\
\hline No & 39 & 79.6 \\
\hline \multicolumn{3}{|c|}{ Does your clinic/hospital provide low vision services? } \\
\hline Yes & 11 & 22.4 \\
\hline No & 38 & 77.6 \\
\hline \multicolumn{3}{|c|}{ Do you know any facility in Saudi Arabia where low vision services are provided? } \\
\hline Yes & 36 & 73.5 \\
\hline No & 13 & 26.5 \\
\hline \multicolumn{3}{|c|}{ Where do you refer patients with low vision? } \\
\hline I do not refer & 12 & 24.4 \\
\hline Tertiary hospital (including KKESH*) & 26 & 53.2 \\
\hline Qassim University & 1 & 2.0 \\
\hline Private low vision clinic & 9 & 18.4 \\
\hline No response & 1 & 2.0 \\
\hline
\end{tabular}

*KKESH = King Khaled Eye Specialist Hospital.

Table 6. Univariate analysis of factors influencing practice.

\begin{tabular}{|c|c|c|c|}
\hline \multicolumn{3}{|l|}{$\begin{array}{c}\text { Characteristics } \\
\end{array}$} & \multirow[t]{2}{*}{ p-value } \\
\hline Have you ever encountered a patient with low vision? & Yes $(\mathrm{n}, \%)$ & No $(\mathrm{n}, \%)$ & \\
\hline \multicolumn{4}{|l|}{ Gender } \\
\hline Male & $27(81.82)$ & $6(18.18)$ & \multirow[t]{2}{*}{0.099} \\
\hline Female & $13(100.00)$ & $0(0.00)$ & \\
\hline \multicolumn{4}{|l|}{ Mode of practice } \\
\hline Government & $21(87.50)$ & $3(12.50)$ & \multirow[t]{3}{*}{0.628} \\
\hline University Eye Clinic & $3(100.00)$ & $0(0.00)$ & \\
\hline Private & $17(80.95)$ & $4(19.05)$ & \\
\hline \multicolumn{3}{|c|}{ Place where the respondent had optometry training } & \\
\hline Inside Saudi Arabia & $29(80.56)$ & $7(19.44)$ & \multirow[t]{2}{*}{0.113} \\
\hline Outside Saudi Arabia & $11(100.00)$ & $0(0.00)$ & \\
\hline Low vision training after graduation & & & \\
\hline
\end{tabular}


(Table $\square$ ) contd.....

\begin{tabular}{|c|c|c|c|}
\hline \multicolumn{3}{|c|}{ Characteristics } & p-value \\
\hline Yes & $21(91.30)$ & $2(8.70)$ & \multirow[t]{2}{*}{0.268} \\
\hline No & $20(80.00)$ & $5(20.00)$ & \\
\hline \multicolumn{4}{|l|}{ Years in practice } \\
\hline$<10$ years & $26(83.87)$ & $5(16.13)$ & \multirow[t]{2}{*}{0.741} \\
\hline$>10$ years & $14(87.50)$ & $2(12.50)$ & \\
\hline \multicolumn{4}{|c|}{ Do you provide low vision services in your practice? } \\
\hline \multicolumn{4}{|l|}{ Gender } \\
\hline Male & $7(20.59)$ & $27(79.41)$ & \multirow[t]{2}{*}{0.962} \\
\hline Female & $3(20.00)$ & $12(80.00)$ & \\
\hline \multicolumn{4}{|l|}{ Mode of practice } \\
\hline Government & $5(20.00)$ & $20(80.00)$ & \multirow[t]{3}{*}{0.109} \\
\hline University Eye Clinic & $2(66.67)$ & $1(33.33)$ & \\
\hline Private & $3(14.29)$ & $18(85.71)$ & \\
\hline \multicolumn{4}{|c|}{ Place where the respondent had optometry training } \\
\hline Inside Saudi Arabia & $7(19.92)$ & $30(81.08)$ & \multirow[t]{2}{*}{0.549} \\
\hline Outside Saudi Arabia & $3(27.27)$ & $8(72.73)$ & \\
\hline \multicolumn{4}{|c|}{ Low vision training after graduation } \\
\hline Yes & $7(29.17)$ & $17(70.83)$ & \multirow[t]{2}{*}{0.136} \\
\hline No & $3(12.00)$ & $22(88.00)$ & \\
\hline \multicolumn{4}{|l|}{ Years in practice } \\
\hline$<10$ years & $3(9.68)$ & $28(90.32)$ & \multirow[t]{2}{*}{$0.010^{*}$} \\
\hline$>10$ years & $7(41.18)$ & $10(58.82$ & \\
\hline \multicolumn{4}{|c|}{ Do you know where low vow vision service is provided in Saudi Arabia? } \\
\hline \multicolumn{4}{|c|}{ Gender } \\
\hline Male & $25(73.53)$ & $9(26.47)$ & \multirow[t]{2}{*}{0.989} \\
\hline Female & $11(73.33)$ & $4(26.67)$ & \\
\hline \multicolumn{4}{|c|}{ Mode of practice } \\
\hline Government & $22(88.00)$ & $3(12.00)$ & \multirow[t]{3}{*}{0.059} \\
\hline University Eye Clinic & $2(66.67)$ & $1(33.33)$ & \\
\hline Private & $12(57.14)$ & $9(42.86)$ & \\
\hline Place where the responde & & & \\
\hline Inside Saudi Arabia & $32(86.49)$ & $5(13.51)$ & $<0.0001^{*}$ \\
\hline Outside Saudi Arabia & $3(27.27)$ & $8(72.73)$ & \\
\hline Low vision trainin & & & \\
\hline Yes & $20(83.33)$ & $4(16.67)$ & 0.125 \\
\hline No & $16(64.00)$ & $9(36.00)$ & \\
\hline Yes & & & \\
\hline$<10$ years & $22(70.97)$ & $9(29.03)$ & 0.384 \\
\hline$>10$ years & $14(82.35)$ & $3(17.65)$ & \\
\hline Do you refer low & & & \\
\hline Gender & & & \\
\hline Male & $25(75.76)$ & $8(24.24)$ & 0.857 \\
\hline Female & $11(73.33)$ & $4(26.67)$ & \\
\hline Mode of practice & & & \\
\hline Government & $18(72.00)$ & $7(28.00)$ & 0.553 \\
\hline University Eye Clinic & $1(50.00)$ & $1(50.00)$ & \\
\hline Private & $17(80.95)$ & $4(19.05)$ & \\
\hline Place where the responde & & & \\
\hline Inside Saudi Arabia & $27(72.97)$ & $10(27.03)$ & 0.651 \\
\hline Outside Saudi Arabia & $8(80.00)$ & $2(20.00)$ & \\
\hline Low vision training after grac & & & \\
\hline Yes & $19(82.61)$ & $4(17.39)$ & 0.243 \\
\hline No & $17(68.00)$ & $8(32.00)$ & \\
\hline Years in practice & & & \\
\hline
\end{tabular}


(Table $\square$ ) contd.....

\begin{tabular}{|c|c|c|c|}
\hline \multicolumn{3}{|c|}{ Characteristics } & p-value \\
\hline$<10$ years & $26(83.87)$ & $5(16.13)$ & \multirow[t]{2}{*}{$0.040 *$} \\
\hline$>10$ years & $9(56.25)$ & $7(43.75)$ & \\
\hline
\end{tabular}

Referral to a low vision clinic was the major action taken by respondents who do not provide low vision services (42.1\%), followed by counselling (27.4\%). Table 7 shows the action taken by respondents who do not provide low vision services.

Table 7. Action taken for patients with low vision.

\begin{tabular}{|c|c|c|}
\hline Action taken & Frequency & Percentage \\
\hline Refer to Low vision clinic & 40 & 42.1 \\
\hline Counsel them on the condition & 26 & 27.4 \\
\hline Go abroad for treatment & 13 & 13.7 \\
\hline Buy hand magnifier & 8 & 8.4 \\
\hline Change job if vision is affecting your job & 4 & 4.2 \\
\hline Seek spiritual help (prayer) & 3 & 3.2 \\
\hline Referred to school for the blind & 1 & 1.0 \\
\hline Total* & $\mathbf{9 5}$ & $\mathbf{1 0 0 . 0}$ \\
\hline
\end{tabular}

*Total exceeded the number of respondents because of multiple responses as each respondent was required to indicate all the actions taken.

\subsection{Barriers}

The barriers reported to militate against the practice of low vision included insufficient training (39.2\%), unavailability of low vision devices locally (24.7\%), amongst others (Table 8 ).

Table 8. Barriers to the provision of low vision services.

\begin{tabular}{|c|c|c|}
\hline Barriers & Frequency & Percentage \\
\hline Insufficient training & 38 & 39.2 \\
\hline Unavailability of low vision devices & 24 & 24.7 \\
\hline Low vision practice is too cumbersome & 8 & 8.2 \\
\hline $\begin{array}{c}\text { Children with low vision do better when they } \\
\text { are sent to the school for the blind }\end{array}$ & 8 & 8.2 \\
\hline $\begin{array}{c}\text { Low vision practice is not financially } \\
\text { rewarding }\end{array}$ & 7 & 7.2 \\
\hline There are too many calculations & 7 & 7.2 \\
\hline Low vision device does not help much & 5 & 5.2 \\
\hline Total* & $\mathbf{9 7}$ & $\mathbf{1 0 0 . 0}$ \\
\hline
\end{tabular}

*Total exceeded the number of respondents because of multiple responses as each respondent was required to indicate all the actions taken.

\section{DISCUSSION}

Given their curriculum (which has a strong base in optics and vision science) [21] and job specification, optometrists are better suited to provide clinical low vision services in KSA. To access optometrists' readiness to provide low vision services in $\mathrm{KSA}$, it was imperative to investigate their knowledge, attitudes, and practices (KAP) of low vision services. From a survey of available database, this appears to be the first study investigating the KAP of optometrists in Saudi Arabia. A study that explored the state of low vision had used a 7-item questionnaire to determine the personnel who provide low vision services in randomly selected eye care facilities in Riyadh [12].

The majority of the respondents do not have accurate knowledge of the definition of low vision in terms of visual acuity $(73.5 \%)$ and whether the low vision is considered to be present after medical, surgical, and optical correction (61.2\%). Respondents in government practices were significantly more likely to have correct knowledge of low vision in terms of visual acuity $(\mathrm{p}=0.023)$. This difference in knowledge could reflect the fact that the majority of optometrists in private practices provide refractive error services using spectacle and contact lenses. Although this is the case for optometrists in government hospitals, these practice in a multidisciplinary setting and therefore may have more interactions with a variety of cases, including patients with low vision. The proportion of those who knew the WHO definition of low vision was similar to the results for Indian eye care professionals [22]. This inappropriate characterization of low vision could lead to poor referral practices. Those who do not have low vision as per WHO definition $[1,2]$ will be referred for services or late referral of those with low vision. Using different visual acuity cut-off points to define low vision has been reported to influence referral patterns by Australian ophthalmologists [23].

Furthermore, respondents have a poor attitude to low vision practice. The majority of respondents held the opinion that low vision practice is time-consuming and not profitable and that optical low vision devices are expensive. Not having low vision specialists and expensive low vision devices have previously been cited as reasons for not providing low vision services in Riyadh [12]. Financial non-viability, the huge cost of low vision services, and time to conduct low vision services have been identified as barriers to low vision services among Canadian optometrists [21]. Over the period since Alotaibi's 2006 study, some Saudi optometrists have undertaken graduate training in low vision care. This held opinion has the effect of dissuading optometrists from providing low vision services. Low vision practice being time-consuming and not lucrative were among the factors identified as perceived barriers to the provision of low vision service among ophthalmologists in Nigeria [24]. The present study showed that nearly $43 \%$ of the respondents are in private practice. These private practices operate to maximize profit and are therefore less likely to engage in low vision practice, which is perceived to be less profitable. Although the government of Saudi Arabia provides health insurance coverage for all her citizens and public service workers, the insurance does not cover spectacle lens correction for refractive errors and optical low vision devices for people with low vision. These factors will further limit access to low vision services in Saudi Arabia. Despite these poor attitudes to low vision services, over $90 \%$ of respondents believed that patients with low vision are not blind and that something can be done to assist them.

Although over $83.7 \%$ of respondents have had an encounter with patients with low vision, $24.4 \%$ of respondents do not refer low vision patients for low vision services. This included $10(20.4 \%)$ who provide low vision services. Respondents who have been in practice for more than 10 years were more likely to provide low vision services than those who have been in practice for less than 10 years $(p=0.010)$, and 
were also less likely to refer patients for low vision services ( $p$ $=0.040$ ). This is probably because, having been in practice for that long, they may have developed skills to provide low vision services. It should be remarked, though, that the present study did not enquire about the nature of low vision services provided. Anecdotal evidence has shown that patients with low vision are usually given handheld magnifiers without appropriate low vision evaluation. Furthermore, optometrists trained outside of Saudi Arabia where less likely to know facilities where low vision services are provided $(\mathrm{p}<0.0001)$. The aggregate of these factors could potentially impact the provision of low vision services in Saudi Arabia.

The study also indicated that respondents who do not provide low vision services offer different courses of action to patients with low vision when they present (Table 7). Though $55.8 \%$ would advise a referral (locally or abroad), it is worthy to note that patients are counseled to take action such as changing jobs, seeking spiritual help, or buying a hand magnifier without appropriate recommendation as to the utility and magnification of the commercially off-the-shelf magnifier. These observations further underscore the fact that optometrists in KSA do not offer appropriate assistance to people with low vision.

Beyond the poor knowledge, attitudes, and practices regarding low vision services, respondents also indicated a number of barriers to their ability to provide low vision services (Table 8). The major barrier identified by respondents is insufficient training (39.2\%). Lack of or insufficient training have been reported to be a major barrier hindering eye care professionals from providing low vision services [23 - 25]. Wrong perceptions about low vision practice were also identified as barriers. These included perceptions that children with low vision benefit from school for the blind, low vision practice is not financially rewarding, and that low vision device does not help people with a low vision much. Several studies have reported the barriers faced by eye care professionals in providing care and referral services for people with low vision [22 - 27]. Some of the barriers related to inadequate training in low vision and poor knowledge and awareness of low vision, and the impact of low vision devices on patients with low vision.

That optometrists in this study and other practitioners in other studies indicated that low vision device does not help patients with low vision much may relate to the fact that clinicians use improvement in clinical measures of visual functions as a yardstick for success in low vision service for patients. Studies have demonstrated the utility of patients' perspectives to evaluate the impact of low vision devices [28, 29]. These studies indicated that optical low vision devices significantly improve the quality of life of patients with low vision despite inapparent improvement in visual acuity or other clinical measures of visual function.

In applying the result from this study, the following limitations to the study should be noted. Firstly the few respondents to this study could affect the generalization of the result to the general population of optometrists in Saudi Arabia. The results, however, provide a peek into the knowledge, attitudes, and practices of optometrists in Saudi Arabia to low vision services. Secondly, being a questionnaire survey, the authors do not have any means of verifying the response of the study respondents.

The poor knowledge, attitudes, and practices of optometrists in Saudi Arabia regarding low vision coupled with the reported barriers (some of which stem from the poor perception of low vision) could affect the provision of low vision services. To address this problem, it is suggested that the low vision course offered at the doctor of optometry (OD) level be reviewed. The review should, among others, address the wrong perception held by optometrists regarding low vision. While this suggestion will focus on those in training, programs aimed at re-educating practicing optometrists to address the knowledge, attitudes, practices, and issues identified in this study should be undertaken for practicing optometrists in Saudi Arabia.

\section{CONCLUSION}

The study identified poor knowledge and attitude towards the practice of low vision amongst optometrists in Saudi Arabia as well as identified a number of barriers militating against the practice of low vision amongst study respondents.

\section{ETHICS APPROVAL AND CONSENT TO PARTICIPATE}

This study was approved by the Research Committee of College of Applied Medical Sciences, Qassim university with approval no. RC/CAMS/19-04-Q-001.

\section{HUMAN AND ANIMAL RIGHTS}

No Animals were used in this research. All human research procedures were followed in accordance with the ethical standards of the committee responsible for human experimentation (institutional and national), and with the Helsinki Declaration of 1975, as revised in 2013.

\section{CONSENT FOR PUBLICATION}

Each participant gave informed consent before filling out the online questionnaire. Being a questionnaire survey, the study posed very minimal risk to study participants; however, the responses were anonymized to preserve the confidentiality of the respondents.

\section{AVAILABILITY OF DATA AND MATERIALS}

The data supporting the findings of the article is available from corresponding author [G.O.O] upon reasonable request.

\section{FUNDING}

This study was carried out as part of the research responsibilities of the authors, who are employees of the Department of Optometry, College of Applied Medical Sciences, Qassim University.

\section{CONFLICT OF INTEREST}

The authors declare no conflict of interest, financial or otherwise. 


\section{ACKNOWLEDGEMENTS}

Declared none.

\section{REFERENCES}

[1] World Health Organization. International statistical classification of diseases, injuries and causes of death, tenth revision. Geneva 1993.

[2] WHO. Management of low vision in children. Geneva: World Health Organization 1992. Contract No.: WHO/PBL/93.27.

[3] Tabbara KF, Ross-Degnan D. Blindness in Saudi Arabia. JAMA 1986; 255(24): 3378-84

[http://dx.doi.org/10.1001/jama.1986.03370240048035] [PMID: 3712697]

[4] Al-Shaaln FF, Bakrman MA, Ibrahim AM, Aljoudi AS. Prevalence and causes of visual impairment among Saudi adults attending primary health care centers in northern Saudi Arabia. Ann Saudi Med 2011; 31(5): 473-80

[http://dx.doi.org/10.4103/0256-4947.84624] [PMID: 21911984]

[5] Al Faran MF, Al-Rajhi AA, Al-Omar OM, Al-Ghamdi SA, Jabak M. Prevalence and causes of visual impairment and blindness in the south western region of Saudi Arabia. Int Ophthalmol 1993; 17(3): 161-5. [http://dx.doi.org/10.1007/BF00942931] [PMID: 8262716]

[6] Al Ghamdi AH, Rabiu M, Hajar S, Yorston D, Kuper H, Polack S. Rapid assessment of avoidable blindness and diabetic retinopathy in Taif, Saudi Arabia. Br J Ophthalmol 2012; 96(9): 1168-72. [http://dx.doi.org/10.1136/bjophthalmol-2012-301874] [PMID: 22790436]

[7] Al-Ghamdi AS. Adults visual impairment and blindness - An overview of prevalence and causes in Saudi Arabia. Saudi J Ophthalmol 2019; 33(4): 374-81.

[http://dx.doi.org/10.1016/j.sjopt.2019.10.001] [PMID: 31920448]

[8] Hajar S, Al Hazmi A, Wasli M, Mousa A, Rabiu M. Prevalence and causes of blindness and diabetic retinopathy in Southern Saudi Arabia. Saudi Med J 2015; 36(4): 449-55.

[http://dx.doi.org/10.15537/smj.2015.4.10371] [PMID: 25828282]

[9] Parrey MU, Alswelmi FK. Prevalence and causes of visual impairment among Saudi adults. Pak J Med Sci 2017; 33(1): 167-71

[http://dx.doi.org/10.12669/pjms.331.11871] [PMID: 28367193]

[10] Tabbara KF, El-Sheikh HF, Shawaf SS. Pattern of childhood blindness at a referral center in Saudi Arabia. Ann Saudi Med 2005; 25(1): $18-21$.

[http://dx.doi.org/10.5144/0256-4947.2005.18] [PMID: 15822489]

[11] Al Faran MF. Prevalence of ocular disorders among schoolboys in five villages in Al-Baha region. Ann Saudi Med 1992; 12(1): 3-7. [http://dx.doi.org/10.5144/0256-4947.1992.3] [PMID: 17589123]

[12] Alotaibi AZ. The state of low vision services in Saudi Arabia. Clin Exp Med Lett 2006; 47(4): 215-8.

[13] Al Motowa S, Khandekar R, Al-Towerki A. Resources for eye care at secondary and tertiary level government institutions in Saudi Arabia. Middle East Afr J Ophthalmol 2014; 21(2): 142-6. [http://dx.doi.org/10.4103/0974-9233.129761] [PMID: 24791105

[14] Aldebasi YH, Ahmed MI, Monaco WA. Are optometrists necessary in primary health care centres in Saudi Arabia? African Vision and Eye
Health 2018; 77(1)

[http://dx.doi.org/10.4102/aveh.v77i1.402]

[15] Raman U. Human resources for eye care: changing the way we think. Commun Eye Health 2009; 22(69): 12

[PMID: 19506715]

[16] Chiang PP-C, O’Connor PM, Le Mesurier RT, Keeffe JE. A global survey of low vision service provision. Ophthalmic Epidemiol 2011; 18(3): 109-21.

[http://dx.doi.org/10.3109/09286586.2011.560745] [PMID: 21609239]

[17] Chiang PP-C, Keeffe JE. Improving access to low vision services. Community Eye Health 2012; 25(77): 15.

[PMID: 22879698]

[18] Andrade C, Menon V, Ameen S, Kumar Praharaj S. Designing and conducting knowledge, attitude, and practice surveys in psychiatry: Practical guidance. Indian J Psychol Med 2020; 42(5): 478-81. [http://dx.doi.org/10.1177/0253717620946111] [PMID: 33414597]

[19] Jacobsen KH. Introduction to health research methods. Burlington, MA: Jones \& Bartlett Publishers 2016.

[20] Health Statistics Annual Book. Riyadh 2018.

[21] Lam HYN. Low vision service provision by optometrists: A nationwide survey. MSc thesis University of Waterloo. 2013.

[22] Jose J, Thomas J, Bhakat P, Krithica S. Awareness, knowledge, and barriers to low vision services among eye care practitioners. Oman J Ophthalmol 2016; 9(1): 37-43.

[http://dx.doi.org/10.4103/0974-620X.176099] [PMID: 27013827]

[23] Keeffe JE, Lovie-Kitchin JE, Taylor HR. Referral to low vision services by ophthalmologists. Aust N Z J Ophthalmol 1996; 24(3): 207-14.

[http://dx.doi.org/10.1111/j.1442-9071.1996.tb01582.x] [PMID: 8913122]

[24] Okoye OI, Aghaji AE, Umeh RE, Nwagbo D F E, Chuku A. Barriers to provision of clinical low-vision services among ophthalmologists in Nigeria. Vis Impair Res 2007; 9(1): 11-7.

[http://dx.doi.org/10.1080/13882350701198702]

[25] Lim HY, O'Connor PM, Keeffe JE. Low vision services provided by optometrists in Victoria, Australia. Clin Exp Optom 2008; 91(2): 177-82.

[http://dx.doi.org/10.1111/j.1444-0938.2007.00214.x] [PMID: 18271781]

[26] Adam R, Pickering D. Where are all the clients? Barriers to referral to low vision rehabilitation. Vis Impair Res 2007; 9: 45-50. [http://dx.doi.org/10.1080/13882350701481033]

27] Khan SA, Shamanna B, Nuthethi R. Perceived barriers to the provision of low vision services among ophthalmologists in India. Indian J Ophthalmol 2005; 53(1): 69-75.

[http://dx.doi.org/10.4103/0301-4738.15293] [PMID: 15829755]

[28] Raasch TW, Leat SJ, Kleinstein RN, Bullimore MA, Cutter GR. Evaluating the value of low-vision services. J Am Optom Assoc 1997; 68(5): 287-95.

[PMID: 9170794]

[29] Ovenseri Ogbomo GO, Osafo-Agyei H, Akpalaba REU, Addy J, Ovenseri EO. Impact of low vision services on the quality of life of low vision patients in Ghana. Afr Vision Eye Health 2016; 75(1): a19. [http://dx.doi.org/10.4102/aveh.v75i1.19]

C) 2021 Ovenseri-Ogbomo and Alghamdi.

This is an open access article distributed under the terms of the Creative Commons Attribution 4.0 International Public License (CC-BY 4.0), a copy of which is available at: (https://creativecommons.org/licenses/by/4.0/legalcode). This license permits unrestricted use, distribution, and reproduction in any medium, provided the original author and source are credited. 